\title{
Demonstration of complex dynamics of light interaction with liquid crystals for educational purposes
}

Patrick LiKamWa, M. Soileau, Nelson Tabiryan, Cesare Umeton, Boris Zeldovich

Patrick LiKamWa, M. J. Soileau, Nelson V. Tabiryan, Cesare Umeton, Boris Ya. Zeldovich, "Demonstration of complex dynamics of light interaction with liquid crystals for educational purposes," Proc. SPIE 2525, 1995 International Conference on Education in Optics, (13 October 1995); doi:

$10.1117 / 12.224055$

Event: SPIE's 1995 International Symposium on Optical Science, Engineering, and Instrumentation, 1995, San Diego, CA, United States 
Demonstration of complex dynamics of light interaction with liquid crystals for educational purposes

\author{
P. LiKamWa, M.J. Soileau, N.V. Tabiryan, C. Umeton*, B.Ya. Zel'dovich \\ Center for Research and Education in Optics and Lasers (CREOL), University of Central Florida, \\ 12424 Research Parkway, Suite 400, Orlando, FL 32826-3271, USA \\ *Department of Physics, University of Calabria, 87036 Arcavacata di Rende (CS), Italy
}

\begin{abstract}
Investigations of interaction of laser beams with liquid crystals reveal new fundamental features of nonlinear dynamic systems. Those features are connected with the giant values of optical nonlinearity, multitude of control parameters, specific properties of light propagation in highly anisotropic and inhomogeneous media, and multidimensional (vector) character of light-induced modulations of liquid crystals. Exceptional opportunities to visualize complex spatio-temporal phenomena in real time, in simple experimental situations, and with utilization of low-power lasers and thin layers of materials, provide a great potential for the design of low-cost and portable set-ups for modeling, demonstrations, and general educational purposes.
\end{abstract}

Key words: education, nonlinear dynamics, pattern formation, complex processes, liquid crystals

\title{
1. COMPLEXITY OF NATURE AND THE PROBLEM OF ITS EXPLORATION
}

Unpredictability, oscillations, instabilities in time and in space, jumps and hysteresis are common to most of natural processes. Wonderful achievement of the recent years have refined the methods of describing complex phenomena, and have established the terms and parameters to characterize them ${ }^{1}$. Most important consequences of these investigations are the concepts of deterministic chaos and spontaneous pattern formation. In the first case, the systems, which are described by nonlinearly coupled deterministic equations, exhibit chaotic behavior in time. In the second case, the homogeneity of a system breaks down, forming spatial patterns of self-organization. The "nonlinear science" possesses not only descriptive, but also predictive power, relating the most intricate features of a process to the number of control parameters, and the parameters, describing the state of the system ${ }^{2}$. Thus the complexity of natural processes is due to the multitude of control parameters, multitude of parameters describing the state of a system and it is born by the nonlinear character of interactions.

Description and characterization of complex phenomena is of vital interest for many disciplines. Learning the principles, understanding, and describing nonlinear phenomena, has become nowadays an essential attribute of successful technological developments, scientific research. It influences the philosophy of science as a whole. It is a highly complicated problem, to carry out investigation of fundamental properties of complex phenomena. First of all, many of them (like weather), "are given and controlled by God," and leave minor opportunities for experimentation. Second, most of complex phenomena hardly allow quantitative characterization, precise measurements, and effective control.

Physical systems are among the simplest ones for realization and investigations of complex phenomena, since they provide the opportunity to control the system and the character of interactions, determining its state.

While development of fundamental investigations is correlated with the possibility of interactive observations and experimentation, the necessity to teach to describe and characterize the principles of complex phenomena, put forward even more severe requirements. First, the model systems should be comprehensible, assuming basic knowledge only. Second, the experimental set-ups should be 
affordable for the budget and space of educational institutions. Experimental situations should be easily modified for different demonstrations, be safe and combine education and entertainment into a single process, since learning is, to a large extenth, gained experience.

It is very hard, however, to achieve these goals with conventional physical systems. For example, modeling through electronic circuits is not visual and requires very special knowledge. Phenomena like turbulent motion in liquids, though simple for experimental realizations, allow rather limited opportunities for manipulations. It is even more difficult to deal with chemical reactions, like Zhabotinskii-Belousov's process.

The stage for research and education to complex phenomena has thus been surrendered to computers, which can be programmed to play any scenario of turbulence and chaos. The value of these nice "movies" about strange attractors and pattern generation would be much higher, if they accompany physical demonstrations.

\section{LASER BEAM INTERACTION WITH MATTER AS A SOURCE OF COMPLEX PHENOMENA AND A POWERFUL TOOL FOR DEMONSTRATIONS}

Recent years proved that nonlinear optical phenomena provide, perhaps, the most striking "pictures" of complex behavior ${ }^{3-4}$. Moreover, the "dry hydrodynamics," as spatio-temporal processes in nonlinear optics are sometimes termed, ensure the largest variety of experimental conditions, best control of the situation, and most precise registration of observation results. Description and visualization of complex phenomena, presented in scientific literature, ultimately suggest, that nonlinear optics is the most suited and comprehensible candidate for modeling and demonstrations for educational purposes.

However, conventional nonlinear optical experiments still encounter the problem of utilization of either high power expensive lasers operating in pulsed regime, or very low-temperatures and complicated registration schemes. Besides, the parameter of the material being influenced by a laser beam is usually a scalar quantity (like temperature or density of charges), putting additional restrictions to the effectiveness of control.

Luckily, there are materials, capable of exhibiting the widest spectrum of complex phenomena with inexpensive, low-power, continuous wave small lasers, with slow dynamics of processes, taking place in the temporal range of seconds and minutes, and at room temperature. These materials are liquid crystals (LC) ${ }^{5}$. They are available commercially in huge variety, due to the developments of LC-display industry. They are cheap, are used in very thin layers (less than $100 \mu \mathrm{m}$ thickness) and in extremely small quantities to prepare a "monodomain" LC-cells for experimentation.

\section{LIQUID CRYSTALS AS THE IDEAL MEDIA FOR NONLINEAR-OPTICAL STUDY OF COMPLEX PHENOMENA}

The specific mechanism of interaction of the laser radiation with LC consists in collective reorientation of the LC-molecules by the electric field of the light, Fig. 1.

Being similar to the LC-reorientation, induced with a dc-voltage in LC-displays, this is, however, a fundamentally new physical phenomenon. It has been under intense investigations during the last about 15 years, exhibiting remarkable richness of unique features, even with the simplest, nematic type of $\mathrm{LC}^{6,7}$.

Unlike solid crystals, reorientation of the optical axis of LC does not require rotation of the crystal as a whole, and is not correlated with macroscopic motion of mass. The forces behind the orientation of LC (orientation of LC's optical axis) are rather weak, and can be overcome with radiation intensities as low as $100 \mathrm{~W} / \mathrm{cm}^{2}$, easily achievable with laser power $10-50 \mathrm{~mW}$. Reorientation of LC influences strongly the 
propagation properties of light, since the optical anisotropy of LC is as high (and even higher), as for many solid crystals praised for high birefringence.

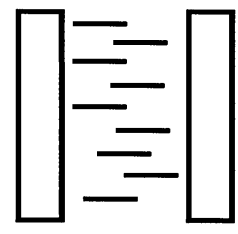

a

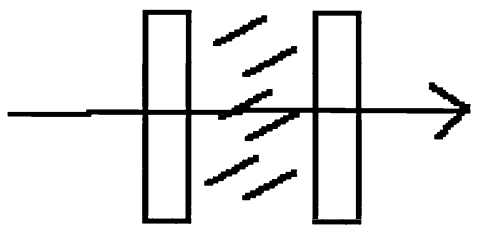

b

Fig.1. Schematic representation of reorientation of molecules of a LC induced by laser radiation: a) LC is sandwitched between transparent glass plates which are treated to align the molecules perpendicular to the plates; b) incident laser radiation exerts a torque on molecules yielding a tilted orientation.

Thus the nonlinear optical constants of LC, characterizing the strength of interaction of laser radiation with LC, turn to be billion times larger than for other liquids and for the most of solid crystals operating at normal conditions. The parameter of LC, which is modulated with the action of a laser beam, is the orientation of the optical axis, i.e. it is a vector. This gives rise to a whole spectrum of new possibilities, compared to scalar optical nonlinearities. Moreover, orientational nonlinear optical phenomena in LC can be easily controlled by external fields, like a dc electric field, temperature gradients, etc. They have nonresonant character, and can effectively take place for any wavelength of laser radiation. Besides the above mentioned features, LC offer an exceptional opportunity to demonstrate and study fundamental properties of light propagation and interaction in highly anisotropic and strongly inhomogeneous media.

The following phenomena have been observed so far, and can make a basis for a kit of interactive demonstrations, affordable for educational institutions: 1. External self-focusing (aberrational nonlinear lens) ${ }^{6,7} .2$. Threshold phenomena and phase transitions ${ }^{6-8} \cdot 3$. Catastrophes ${ }^{9-11}$. 4. Temporal instabilities from periodic oscillations to stochastic dynamics and strange attractors ${ }^{12}$. 5. Pattern formation a) in feedback systems ${ }^{13-14}$, and b) due to light-induced caustics and interference ${ }^{15}$. 6 . LC light valves for demonstrations of time reversal (phase conjugation) ${ }^{16}$.

One more important phenomenon of fundamental physics, which is strikingly exhibited, when a laser beam crosses a LC-cell, is the fluctuations of the orientation of molecules of LC due to Brownian motion. This motion reveals itself as chaotic wandering of bright spots of light scattered by LC.

It is important to note, that the mentioned and other phenomena can be realized in one and the same system, with straightforward modifications of control parameters: incidence angles, polarization and intensities of laser beams. Stress out that the "intimate" details of nonlinear dynamics of the interaction of laser beams with LC can be just projected to the screen, in the form of high brightness and high contrast annular ring system Fig.2. "Breathing" of that ring pattern is what can be observed with a naked eye, and measured with a usual watch. 


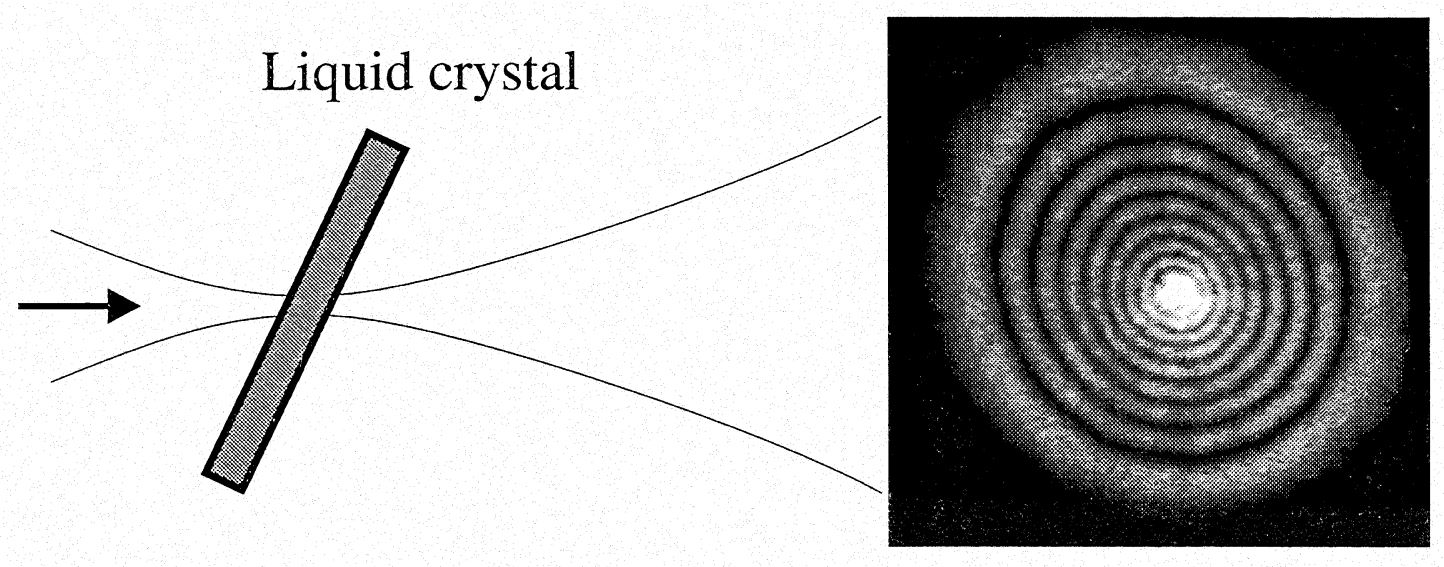

Fig.2. Reorientation of LC induced by a laser beam gives rise to an annular ring pattern with the number of rings being the measure of the reorientation magnitude. Oscillations in the number of rings reveals the dynamics of the reorientation of LC. The divergence angle of the outermost ring can be as large as 45 degree.

Both lasers and LC are essential parts of the present-day technologies, underlying optical communication, information display and processing, measurement, and entertainment systems. Involvement with modern technologies contributes highly to the attractiveness of investigation and demonstration of complex phenomena with the aid of processes, taking place as a result of interaction of laser beams with LC. The pedagogical principles of teaching nonlinear optics, in particular, and complex phenomena, in general, were implemented for several years at the Moscow Institute for Engineering and Physics, Russia, at the University of Calabria, Italy, and were practiced in other educational institutions.

\section{REFERENCES}

1. H.D.I.Aarbanel, R.Brown, J.J.Sidorowich, L.Sh.Tsimring, "The analysis of observed chaotic data in physical systems", Rev. Mod. Phys. 65 (4), 1331-1392, 1993.

2. T.Poston, I.Stewart, "Catastrophe Theory and Its Applications", Pitman, London, 1978.

3. F.T.Arecchi, R.G.Harrison, "Selected papers on optical chaos", SPIE Milestone Series, Vol. MS 75, 1994.

4.N.B.Abraham, W.J.Firth, "Overview of transverse effects in nonlinear-optical systems", $J$. Opt. Soc. Am. B 7 (6), 951-962, 1990.

5. P.G. de Gennes. The Physics of liquid crystals, Clarendon Press, Oxford, 1974.

6. N.V.Tabiryan, A.V.Sukhov and B.A.Zel'dovich," The orientational optical nonlinearity of liquid crystals", Mol. Cryst. Liq. Cryst., Special Topics XIX, 136, 1-140, 1986.

7. I.C.Khoo, S.T.Wu, "Optics and Nonlinear Optics of Liquid Crystals", World Scientific, 1993.

8. B.Ya.Zel'dovich, S.R.Nersisyan, N.V.Tabiryan, "Threshold interaction of extraordinary light waves with nematics", Sov. Phys. JETP, 61 (4), 712-718, 1985.

9. K.E.Asatryan, A.R.Mkrtchyan, S.R.Nersisyan, N.V.Tabiryan, "Catastrophes in orientational interaction of an optical wave with a nematic liquid crystal", Sov. Phys. JETP, 68 (2), 315-318, 1989.

10. E.Santamato, G.Abbate, R.Calselice, P. Maddalena, A.Sasso, "All-optical-field-induced firstorder Freedericksz transition and hysteresis in a nematic film”, Phys. Rev. A, 37 (4), 1375-1377, 1988.

11. K.E.Asatryan, A.R.Mkrtchyan, Yu.A.Reznikov, N.V.Tabiryan, V.B.Vinogradov, "Observation of orientational catastrophes in cholesteric liquid crystals”, Opt. Spectrosc. (USSR), 69 (4), 495-498, 1990. 
12. V.Carbone, G.Cipparrone, C.Versace, R.Bartolino, C.Umeton, N.V.Tabiryan, "Experimental investigation of light induced chaotic dynamics in nematic liquid crystals", Mol. Cryst. Liquid Cryst., 251, 167-180, 1994.

13. R.Macdonald, H.J.Eichler, "Spontaneous optical pattern formation in a nematic liquid crystal with feedback mirror”, Opt. Commun., 89 (2-4), 289-295, 1992.

14. M.Tamburrini, M.Bonavita, S.Wabnitz, E.Santamato, "Hexagonally patterned beam filamentation in a thin liquid-crystal film with a single feedback mirror", Opt. Lett., 18 (11), 855-857, 1993.

15. N.V.Tabiryan, B.Ya.Zel'dovich, M.Kreuzer, T.Vogeler, T.Tschudi, "Higher dimensionality caustics due to competing reorientation of liquid crystal by laser beams", Submitted to J.Opt. Soc. Am., B.

16. O.V.Garibyan, I.N.Komponets, A.V.Parfyonov, N.F.Pilipetskii, V.V.Shkunov, A.N.Sudarkin, A.V.Sukhov, N.V.Tabiryan, A.A.Vasiliev, B.Ya.Zel'dovich, "Optical phase conjugation by microwatt power of reference waves via liquid crystal light valve", Opt. Commun., 38 (1), 67-70, 1981. 